\section{Medical Education During the COVID-19 Pandemic: A Single Institution Experience}

Social distancing to curb the COVID-19 pandemic has caused suspension of classroom teaching in all educational institutions. We implemented a novel online classroom platform at our institute to continue medical education. The program attracted encouraging feedback from the students. It may serve as a model for uninterrupted teaching and training during times of crisis.

Keywords: COVID-19, G Suite for Education, Online classroom.

Published online: May 04, 2020; PII: S097475591600174

$\mathrm{T}$ he novel coronavirus (SAR-CoV-2) pandemic has disrupted medical education worldwide [1]. Most of the medical schools have quickly adapted to the online classes with shifting of live clinical exposure with the virtual one. Some schools also echoed concerns over clinical clerkships and assessment during these times. The COVID-19 pandemic represents a transformation in medicine with the advancement of telehealth, adaptive research protocols, and clinical trials with flexible approaches to achieve solutions [2-4]. We herein share our early initial experience of online training of medical students in the setting of COVID-19.

In wake of impending restrictions, we explored available options for online classes and adopted G Suite for Education using Google Classroom coupled with Google Meet for Video-conferencing (https:// edu.google.com/products/gsuite-for-education/ ?modal_active=none). A schedule was made and messages were sent to students by email and short messaging service (SMS) to join their respective classes. An orientation program was conducted to familiarize the faculty to this platform. A team of trained faculty members was deputed at the lecture venues to assist and troubleshoot technical issues, if any. Additionally, training videos were shared with faculty members.

In order to minimize excessive data usage by students and preventing high screen time, a four-hour teaching schedule, ensuring a judicious mix of lectures and practical demonstrations/case discussions were employed with a break of 10-15 minutes between sessions. To promote student engagement, and to closely replicate laboratory and clinical environment, short videos on lab procedure and case based clinical examination were prepared and shared on the virtual classroom. To make the session interactive, students were encouraged to use chat-box and switch on their microphones, wherever feasible. Assignments were administered through inbuilt plugins.

A questionnaire was prepared and administered via Google forms to the students belonging to different semesters of the MBBS course. The questionnaire was reviewed and validated by the involved faculty members. Participation was voluntary and complete anonymity was ensured. Data was compiled using spreadsheets. Gaussian fit of data was assessed using KolmogorovSmirnov test.

Across four batches from second to eighth semester, 398 medical undergraduate students were enrolled in the classes; 208 provided their responses to the questionnaire, with similar proportion across various semesters (44-61\%). The detailed responses are depicted in Table I. The students, based on their quantitative (Fig. 1) and qualitative feedback, appreciated the online platform. Large number of students had not attended any online classes previously. Majority of the students stated that they were given the opportunity to ask questions $(92.3 \%)$. They believed their interaction with the teacher was better than $(27 \%)$ or as good as $(27.8 \%)$ that during physical classroom. The responses across semesters were also uniformly similar.

Interestingly, innovative solutions have emerged whenever such problems have set in during SARS and MRES outbreaks using telephone and virtual environment [5,6], and other adaptations during COVID-19 [7]. While Moszkowicz, et al. [8] implemented Google Hangouts for a similar purpose but with only 10 students,

Table I Feedback on Online Teaching by Medical Undergraduates $(N=\mathbf{2 0 8})$

\begin{tabular}{lr}
\hline Feedback question & No. (\%) \\
\hline $\begin{array}{l}\text { Previously attended any online classes } \\
\text { Given the opportunity to ask questions during }\end{array}$ & $73(35)$ \\
$\quad$ the e-classes? & $191(92.3)$ \\
The material shared before/after e-classes & \\
$\quad$ was useful & \\
How do you rate your interaction with the teacher & \\
during e-classes? & \\
As good as physical classroom & $58(28.0)$ \\
Better than physical classroom & $58(28.0)$ \\
Poorer than physical classroom & $91(43.9)$ \\
Select the statement that applies best to you: & $106(50.9)$ \\
Physical classes are better than e-classes & $56(26.9)$ \\
E-classes are as good as physical classes & $46(22.1)$ \\
E-classes are better than physical classes &
\end{tabular}

*One student did not reply. 
we conducted concurrent sessions for a large number belonging to four different semesters. Our platform also supported flipped classroom to some extent by providing learning material in advance and promoting student discussion during online sessions [9].

Student feedback revealed some interesting paradox. While appreciative of the platform, nearly $50 \%$ of the students still believed that physical classroom was better than e-classroom. However, the reasons for this perception could not be assessed. The study was based on a small sample of students who have anonymously volunteered to provide feedback. Secondly, we had very short time to implement and hence a well-structured training program for faculty could not be done. This was; however, circumvented to some extent, by the ease and self-explanatory nature of the platform, a short explanatory video and provision of technical support at lecture venues. Furthermore, majority of our teachers got adapted to this forum after taking a couple of classes.

The novelty of the initiative lies in the swift implementation of this program on a large scale both for the students and for faculty members. Another study from India has previously reported using the same platform, but restricted to a single specialty [10]. We believe our early experience can serve as a model for educational institutes looking for continuing medical education in situations that disrupt traditional teaching.

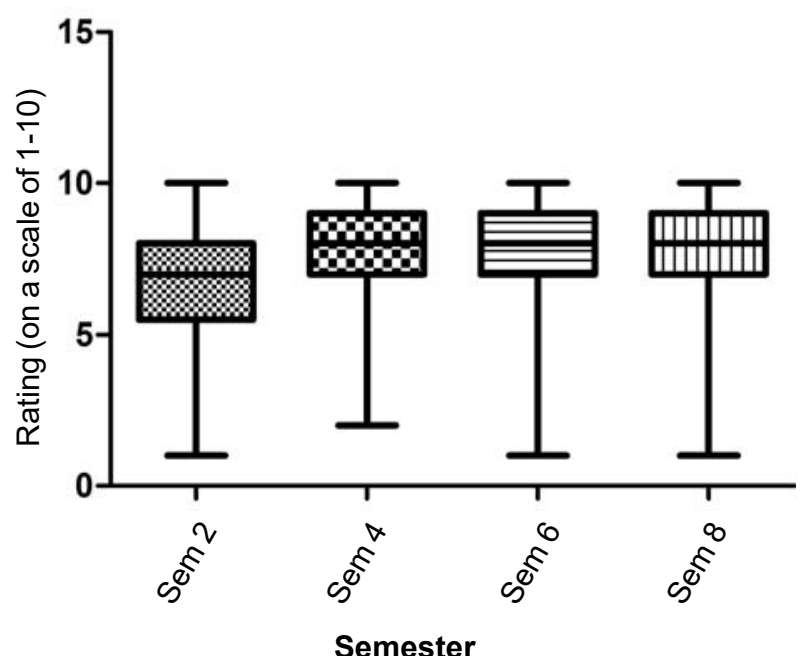

Number of students in II, IV, VI and VIII semesters was 61,47,44 and 56 , respectively.

Fig. 1 Rating of online classes during COVID-19 pandemic by medical graduates of different semesters on a Likert scale of 1-10.
Contributors: All authors have contributed, designed and approved the study.

Funding: None; Competing interest: None stated.

KuldeEp Singh ${ }^{*}$, Shival SrivastaV ${ }^{2}$, Abhishek BHARDWAJ $^{3}$, ABHINAV DIXIT ${ }^{2}$ AND SANJEEV MISRA ${ }^{4}$ Departments of ${ }^{l}$ Pediatrics, ${ }^{2}$ Physiology, ${ }^{3}$ Dermatology and Surgical Oncology 4 All India Institute of Medical Sciences, Jodhpur, Rajasthan, India.

\section{REFERENCES} *kulpra@hotmail.com

1. Pather N, Blyth P, Chapman JA, Dayal MR, Flack NAMS, Fogg QA, et al. Forced disruption of anatomy education in Australia and New Zealand: An acute response to the Covid-19 pandemic. Anat Sci Educ. 2020 Apr 18. Available from https://anatomypubs.onlinelibrary. wiley.com/doi/epdf/10.1002/ase.1968. Accessed April 23, 2020.

2. Rose S. Medical student education in the time of COVID19. JAMA. 2020 Mar 31. Available from: https:// jamanetwork.com/journals/jama/fullarticle/2764138. Accessed April 14, 2020.

3. Liang ZC, Ooi SBS, Wang W. Pandemics and their impact on medical training: Lessons from Singapore. Acad Med. 2020 Apr 17. Available from https://journals.lww.com/ academicmedicine/Abstract/9000/Pandemics_and_ Their_Impact_on_Medical_Training_.97208.aspx. Accessed April 23, 2020.

4. Li L, Xv Q, Yan J. COVID-19: The need for continuous medical education and training. Lancet Respir Med. 2020;8:e23.

5. Patil NG, Yan YCH. SARS and its effect on medical education in Hong Kong. Med Educ. 2003;37:1127-8.

6. Park SW, Jang HW, Choe YH, Lee KS, Ahn YC, Chung MJ, et al. Avoiding student infection during a Middle East respiratory syndrome (MERS) outbreak: A single medical school experience. Korean J Med Educ. 2016;28:209-17.

7. Hodgson JC, Hagan P. Medical education adaptations during a pandemic: transitioning to virtual student support. Med Educ. 2020 April 14. https://onlinelibrary.wiley.com/ doi/abs/10.1111/medu.14177. Accessed April 23, 2020.

8. Moszkowicz D, Duboc H, Dubertret C, Roux D, Bretagnol F. Daily medical education for confined students during COVID-19 pandemic: A simple videoconference solution. Clin Anat. 2020 Apr 6. Available from: https:// onlinelibrary.wiley.com/doi/full/10.1002/ca.23601. Accessed April 23, 2020.

9. Singh K, Mahajan R, Singh T, Gupta P. Flipped classroom: A concept for engaging medical students in learning. Indian Pediatr. 2018;55:507-12.

10. Dash S. Google classroom as a learning management system to teach biochemistry in a medical school. Biochem Mol Biol Educ. 2019;47:404-7. 